\title{
Comparative Prophylactic Administration Effect of Metronidazole, Ceftriaxone Sodium and their Combination on the Tensile Strength of End-to- End Jejunal Anastmotic Site in Dogs
}

\section{Rehan Rafique ${ }^{1,2}$, Muhammad Nadeem Asi ${ }^{2}$, Muhammad Saqib ${ }^{2}$, Muhammad Hammad Hussain ${ }^{2}$, Aqsa Mushtaq ${ }^{1}$, Awais-ur-Rehman Sial' ${ }^{2}$, Hina Afroz ${ }^{1}$ and Muhammad Najiullah Khan ${ }^{1}$}

\author{
${ }^{1}$ Veterinary Research Institute, Zarrar Shaheed Road, Lahore Cantt, Punjab, Pakistan \\ ${ }^{2}$ Department of Clinical Medicine \& Surgery, University of Agriculture Faisalabad, Punjab, Pakistan
}

Correspondence should be addressed to: Rehan Rafique; dr.rehan702@gmail.com

Received 17 May 2013; Accepted 25 June 2013; Published 28 August 2013

Academic Editor: Sun Hee Do

Copyright (c) 2013 Rehan Rafique, Muhammad Nadeem Asi, Muhammad Saqib, Muhammad Hammad Hussain, Aqsa Mushtaq, Awais-ur-Rehman Sial, Hina Afroz and Muhammad Najiullah Khan. Distributed under Creative Commons CC-BY 3.0

\begin{abstract}
Intestinal resections are most commonly performed in dogs and currently it is involved with extensive use of different antibiotics before surgical intervention. Metronidazole and cephalosporins are considered as best choice for prophylactic use in gastrointestinal operations. Therefore, the study is established to reveal the comparative effect of metronidazole, ceftriaxoine sodium and their combination on the tensile strength of jejunual anastomotic site. A total of twenty healthy stray dogs were included in this study. These dogs were randomly divided into four groups, which underwent end to end jejunal ansastmosis. The dogs in Group I (control) were operated for jejunal anstomosis without any antibiotic prophylaxis. The metronidazole alone was administered intravenously to dogs in Group II at the dose rate of $50 \mathrm{mg} / \mathrm{kg}$ while the dogs in Group III were administered with intravenous ceftriaxone sodium at the dose rate of $30 \mathrm{mg} / \mathrm{Kg}$ body weight two hours prior to surgery. The dogs in Group IV were given metronidazole in combination with ceftriaxone sodium by intravenous route at the dose rate of $50 \mathrm{mg} / \mathrm{Kg}$ and $30 \mathrm{mg} / \mathrm{kg}$ respectively two hours before surgical intervention. A significant difference was noted in the tensile strength between the four studied groups $(\mathrm{P}<0.05)$. The study concluded that the combination of ceftriaxone sodium with metronidazole was found to enhance the gain in tensile strength significantly i.e. $47.50 \%$.
\end{abstract}

Keywords: Jejunum, End- to- end anastmosis, Metronidazole, Ceftriaxone sodium, Tensile strength.

Cite this Article as: Rehan Rafique, Muhammad Nadeem Asi, Muhammad Saqib, Muhammad Hammad Hussain, Aqsa Mushtaq, Awais-ur-Rehman Sial, Hina Afroz and Muhammad Najiullah Khan (2013), "Comparative Prophylactic Administration Effect of Metronidazole, Ceftriaxone Sodium and their Combination on the Tensile Strength of End-to-End Jejunal Anastmotic Site in Dogs," International Journal of Veterinary Medicine: Research \& Reports, Vol. 2013 (2013), Article ID 901239, DOI: $10.5171 / 2013.901239$ 


\section{Introduction}

Surgical anastmosis of various parts of intestine is most commonly performed in animals especially in dogs and cats to relive out the irresolvable intestinal obstruction or de-vitalization (Tobias and Ayres, 2006; Gregory, 2003; Rasmussen, 2002). Jejunum is the longest part of small intestine that increases its possible chance to the surgical intervention at some specific location (Dilawar et al., 2011).

Surgical wound infections are frequent complications following gastrointestinal tract surgery. Infections related to surgical wound consequence in the administration of additional antimicrobial agents. It is extensively approved that appropriate antimicrobial prophylaxis is favorable in gastrointestinal surgery that has high risk to intestinal flora (Dellinger et al., 1994).

Prior 1970, the intestinal anastomotic resections were fraught with postoperative infectious complications which occurred in more than $30-50 \%$ of all the surgical approaches to the intestine. Due to recognition of the importance of appropriate prophylactic antimicrobial therapy directed at both aerobic and anaerobic species of bacteria, surgeons have been able to perform single-stage intestinal resections with primary anastomosis routinely. The current century brought the revolution in gastrointestinal surgery, resulting in significant decrease in infectious complications, in animals including dogs with the reduced postoperative complications due to the antibiotic prophylaxis, improved surgical techniques, new anesthesia drugs and protocols as well as both pre \& postsurgical management (Nichols et al., 2005). The prophylactic use of antimicrobial agents is the current standard of care prior to gastrointestinal surgery (Basany et al., 2005).

Recently, pre-operative prophylactic antibiotics became a foundation of therapy, the accurate and judicious administration of antibiotics have become performance measures for the excellence perfection and safe surgical procedures worldwide. As it is obvious from the published literature, to minimize the chances of postoperative infections for the gastrointestinal surgical procedures, the prophylactic use of different antibiotics has become the current standard of care. Metronidazole and ceftriaxone sodium are most commonly administered prophylactic agents for gastrointestinal anastomosis and considered as best choice as compared to other options. Keeping in view the highlighted facts, the purpose of present study was to evaluate the comparative effect of most commonly used prophyactic metronidazole and ceftriaxone sodium on the tensile strength of the jejunal anastmotic segment of dogs.

\section{Material and Methods}

The study comprised of twenty healthy stray dogs that were randomly divided into four groups, with five animals in each group. The studying animals included twelve males and eight females weighing between 14 to 18 kilograms. Each studied group included three male and two female stray dogs. These experimental dogs were identified by collar tags. The dogs of GroupI were kept as control and operated for jejunal anastomosis without administering any prophylactic antibiotic regime. The animals of Group-II were only given prophylactic metronidazole at the dose rate of $50 \mathrm{mg} / \mathrm{Kg}$ body weight intravenously, two hours before surgery. While the GroupIII dogs were administered intravenously with ceftriaxone-sodium alone at the dose rate of $30 \mathrm{mg} / \mathrm{Kg}$, two hours before surgical intervention. The intravenous administration of metronidazole and ceftriaxone-sodium were given in combination to animals of Group-IV at the dose rate of $50 \mathrm{mg} / \mathrm{Kg}$ and $30 \mathrm{mg} / \mathrm{Kg}$ body weight respectively, two hours prior to surgery (Hinchey et al., 1983; Woodfield et al., 2003).

\section{Clinical Examination}

Before experimentation, acclimatizing period of fourteen days was provided to all the dogs, so that they get used to the new environment to minimize stress factors. During this acclimatization period, the dogs

Rehan Rafique, Muhammad Nadeem Asi, Muhammad Saqib, Muhammad Hammad Hussain, Aqsa Mushtaq, Awais-ur-Rehman Sial, Hina Afroz and Muhammad Najiullah Khan (2013), International Journal of

Veterinary Medicine: Research \& Reports, DOI: 10.5171/2013.901239 
were subjected to thorough physical and clinical examination for the evaluation of their health status. Hematological parameters including hemoglobin, hematocrit values, total erythrocyte and leukocyte count were determined by following the methods as described by Jain (1986). Experimental dogs were kept indoor in separate clean stainless steel cages for two weeks pre-operatively to rule out the possibility of any latent or nosocomial infection. Physiological parameters like body temperature, pulse and respiration rates were also recorded daily for one week preoperatively to obtain the baseline data and two weeks following surgical intervention. The experimental dogs were fed on bread and milk. Fresh water was available ad libitum except three hours before surgical intervention and six hours post-operatively.

\section{Surgical Procedure}

Before the administration of thiopentone sodium (general anesthetic agent), each dog from all four groups was premedicated by administering atropinesulphate@ $0.045 \mathrm{mg} / \mathrm{Kg}$ body weight through subcutaneous (S/C) route, half an hour prior to surgical intervention to minimize secretions of the salivary glands and respiratory tract (Plumb, 2008). Thiopentone sodium was administered by the slow vein puncture of cephalic vein, at a dose rate of $25 \mathrm{mg} / \mathrm{Kg}$ body weight. Then laprotomy procedure was performed by following adequate pre-operative measures. Each dog was positioned as $30^{\circ}$ tilted to the horizon in the dorsal recumbency and umbilicus was considered as surgical landmark. Ventral midline incision of about $12 \mathrm{~cm}$ length was made through the skin and subcutaneous tissue with the help of scalpel blade. Then peritoneal cavity was opened carefully by a stab incision and the falciform ligament was resected with scissors. This permitted uniform peritoneal contact at the closure, and minimized any risk of wound dehiscence (Tavakoli et al., 2007). After exposing the abdominal viscera; a part of the jejunum was selected and packed off for resection. Common jejunal and mesenteric arteries supplying to the selected part were isolated and ligated. The jejunum was transected with a scalpel between the crushing and non-crushing forceps along the edges of the crushing forceps. The resected section of the jejunum, with the two crushing forceps, was removed. The ansatomotic site of jejunum was then examined critically for the patency and anastomotic leaks. Suturing in three layers was accomplished for the closure of the abdomen (Weisman et al., 1999; Fossum, 2007).

After the surgical intervention each dog was then observed for recovery and placed back in the dog ward after recovery. Water was offered ad libitum followed by the milk on the postoperative day 2 and a soft diet on day 3 postoperatively. Body temperature, pulse and respiration readings were recorded twice a day for the fourteen days after operation. Each animal was given complete antiseptic dressing once daily. After 14 days, all the dogs were euthanized to find out the effect of treatments on the tensile strength.

\section{Tensile Strength Measurement}

The tensile strength (TS) of the anastomotic site was recorded at day $14^{\text {th }}$ post-operatively with a device Schopper's tensile strength tester No. 114-SC type (Yasuda Seiki Seisakusho, Japan). The instrument is specially designed for measuring the tensile strength of vinyl, rubber, leather, copper-wire, fabric and cord etc. This tester is designed and manufactured in accordance with Schopper's mechanism on the basis of principle of balance for measuring tensile strength. For this purpose, the $6 \mathrm{~cm}$ intestinal segments containing jejunal anastomotic site were cut 14 days after the operation by euthanizing the all dogs. Following washing of the specimen with normal saline, three $1 \times 2 \mathrm{~cm}$ strips containing the anastomotic site in the center and three same sized strips from the un-operated part of the jejunum (as control) were cut. Each piece was clumped in the upper and lower chucks of the Schopper's Tensile Strength Tester leaving $1 \mathrm{~cm}^{2}$ area on the either side of the healing line and subjected to the instrument for the 
measurements. Tensile strength was noted as the force units (grams) indicated by the pointer on the holding scale of the tensile tester when the rupture occurred. Similar strips from the healthy (control strips) intestinal segment of all dogs were also evaluated for their tensile strength. The percent gain in the tensile strength (using means of 3) was calculated by the formula (Booth, 1968).

Gain in the tensile strength $=$ Mean TS of strips with anastomotic site X 100

Mean TS of control strips

\section{Results}

The average gain in the tensile strength of the Group I animals was 26.50 percent. For this group, the results for gain in tensile strength were ranging between 22-31 percent. The lowest gain in tensile strength, in this group, was 22.73 percent. While the highest gain in tensile strength 30.95 percent was recorded in this group. The dogs that were prophylactically administered with metronidazole (Group II) revealed 39.70 percent average gain in the tensile strength. The percentage gain in tensile strength of this group was found to be significantly higher than Group I. The gain in tensile strength of metronidazole treated group was ranging between 37-42 percent. The highest recorded gain in tensile strength was 41.67 percent, while 37.50 percent was the lowest recorded gain in tensile strength. The group of dogs that were prophylactically administered with only ceftriaxone sodium (Group III), the highest average gain in tensile strength 44.83 percent was recorded. The average gain in tensile strength of this group 43.6 percent was significantly higher from Group I and Group II. The gain in tensile strength of this group was ranging between 41-45 percent, with highest gain in tensile strength recorded as 44.83 percent. While 41.67 was the lowest gain in tensile strength of this group. The average gain in tensile strength recorded, for the animals that were prophylactically administered with combination of ceftriaxone sodium and metronidazole (Group IV), was 47.50 percent which is significantly higher than the dogs of Group I, Group II and Group III. The gain in tensile strength of this group was ranging between 44-50 percent with the highest value of gain in tensile strength recorded as 50.0 percent. However, the lowest gain in tensile strength of this group was 44.0 percent.

Table I: Pre-Operative Prophylactic Effect of Treatment Groups on Tensile Strength of Anastomotic Portion of Jejunum

\begin{tabular}{|l|l|}
\hline Experimental Groups & Mean Tensile Strength (\%) \\
\hline \hline Control & $26.5 \pm 3.44$ \\
\hline Metronidazole & $39.7 \pm 1.50$ \\
\hline Ceftriaxone Sodium & $43.6 \pm 1.29$ \\
\hline Ceftriaxone Sodium+ Metronidazole & $47.5 \pm 2.27$ \\
\hline
\end{tabular}

Rehan Rafique, Muhammad Nadeem Asi, Muhammad Saqib, Muhammad Hammad Hussain, Aqsa Mushtaq, Awais-ur-Rehman Sial, Hina Afroz and Muhammad Najiullah Khan (2013), International Journal of

Veterinary Medicine: Research \& Reports, DOI: 10.5171/2013.901239 


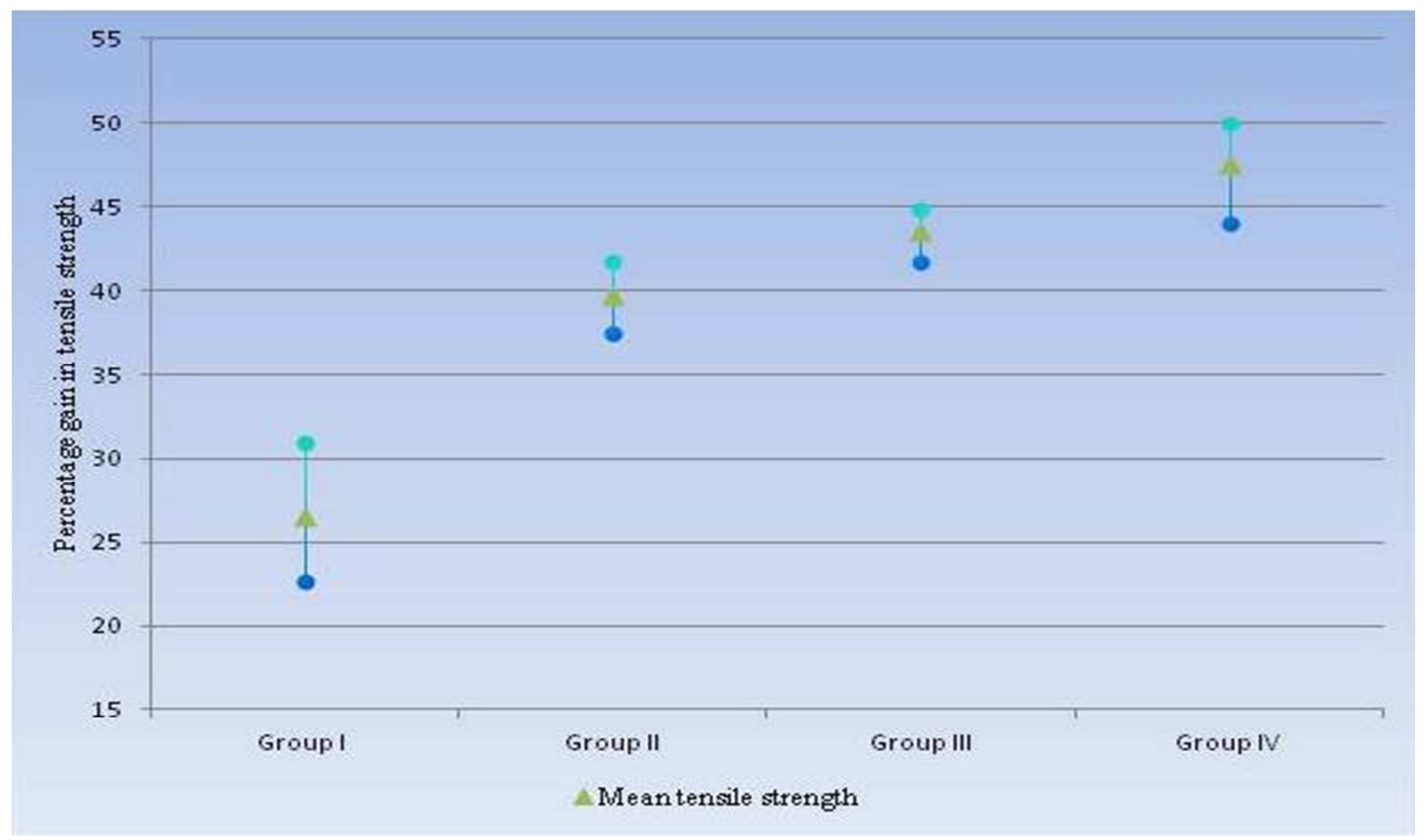

Figure 1: Graphical Representation for the Gain in Tensile Strength of Studied Groups

\section{Statistical Analysis}

The analysis of variance (ANOVA) statistical test was applied to the data obtained from the findings of mean tensile strength of each group and results expressed that among all the four studied groups, a highly significant difference was observed as probability is less than 0.01 (P $<0.01$ ). The highest percentage gain in tensile strength of jejunum $47.5 \pm 2.27$ percent was observed in animals that were treated with combination of Ceftriaxone Sodium and Metronidazole (Group IV). While the lowest percentage gain in tensile strength $26.5 \pm 3.44$ was recorded in animals of control group (Group I).

\section{Discussion}

Restoration of normal tissue strength is among the most accepted and reliable indices of wound healing (Van Winkle, 1969; Athar et al., 1996). In case of intestinal anastomosis, tensile strength is not a function of the length of the incision or of the thickness of the tissue rather, it is determined in term of load applied per unit of cross section area. In the present study, increase in the tensile strength of the anastomosed jejunal segment was measured on day 14 postsurgical intervention following the techniques of Athar et al., (1996). Jejunal anastomotic segments of the dogs that were administered prophylactically with single dose of intravenous ceftriaxone sodium alone (Group III) and in combination with metronidazole (Group IV) had statistically highly significant increments in tensile strength (Table I) as compared to the remaining two regimens i.e. prophylactically single dose intravenous administration of metronidazole alone (Group II) and control group that was not given any prophylactic antibiotic (Group I). Moreover, the jejunal anastomotic segments of the dogs that were administered pre-operatively with single intravenous dose of metronidazole (Group II) also had statistically significant increase in tensile strength (Table I) as compared to control group (Group I). The least gain in tensile strength was observed in control group and it might be related to the

Rehan Rafique, Muhammad Nadeem Asi, Muhammad Saqib, Muhammad Hammad Hussain, Aqsa Mushtaq, Awais-ur-Rehman Sial, Hina Afroz and Muhammad Najiullah Khan (2013), International Journal of

Veterinary Medicine: Research \& Reports, DOI: 10.5171/2013.901239 
presence of infective organisms at the anastomotic site.

In all three treatment groups (Group II, GroupIII and Group IV), the gain in the tensile strength seems to be due to reduction in the colonization of normal and pathogenic micro-organisms, which on getting chance might contaminate the anastomotic wound and ultimately causing post-operative infections. These results concur with those reported by Athar et al., (1996), Hayashi and Wilson (2009), Wang et al., (2003), and Woodfield et al., (2003). Statistically lesser gain in tensile strength recorded in the control animals. It was observed that during study, the animals of this group had suffered from fever for three to four days that's why the gain in the tensile strength was lower than the groups having single dose of intravenous antibiotic prophylaxis. These findings are also inline with those of Athar et al., Cai (1992), Leaper (1994), Lindhagen et al., (1981), Mittermayer et al., (1984) Ono et al., (1990) Parker et al., (1985) Playforth et al., (1988) and Takesue et al., (2000). As for the choice of antimicrobial agents, it is general consensus worldwide that administration of prophylactic antibiotics should be intravenous as single dose preferably 1 hour to 30 minutes before surgery. In our study it is thus not surprising that ceftriaxone sodium in combination with metronidazole has more prophylactic efficacy. This high prophylactic efficacy might be due to specific pharmacokinet profile of these antimicrobials which ensures the bactericidal actions against aerobic and anaerobic pathogenic organisms. Similar results for prophylactic efficacy of ceftriaxone sodium in combination with metronidazole also discussed by Athar et al., (1996), Gravante and Caruso(2009), Lalla (2009), Rau et al., (2000) and Ross et al., (2009).

\section{Acknowledgement}

The authors are grateful to Prof. Dr. Ghulam Muhammad, who shared the valuable information. During this study, their kind and encouraging attitude is highly appreciateable.

\section{References}

Athar, M., Chaudhry, N. I., Shakoor, A. \& Khan, M. A. (1996). "Studies on End-to-End Colonic Anastomosis in the Dog: A Comparison of Techniques," Acta Veterinaria Hungarica, 44, 349-356.

Basany, E. E., Garcia, J. L. S., Cano, M. L., Trujillo, R. L., Ferrer, M. M., Gil, L. A., Vilches, L. A. \& Carrasco, M. A. (2005). "Prospective, Randomised Study on Antibiotic Prophylaxis in Colorectal Surgery. Is It Really Necessary to Use Oral Antibiotics?," International Journal of Colorectal Disease, 20 (10) 542-546.

Booth, J. E. (1968). Principles of Textile Testing: An Introduction to Physical Methods of Testing Textile Fibres, Yarns and Fabrics, Kingsway, London, UK.

Cai, C. J. (1992). "Clinical Study of Prophylactic Use of Gentamicin and Metronidazole in the Surgery of Colorectal Carcinoma," Zhonghua Wai Ke Za Zhi, 30 (4) 237-240.

Dellinger, E. P., Gross, P. A., Barett, I. L., Krause, P. J., Martone, W. J., Mcgowan, J. E., Sweet, K. L. \& Wenzel, R. P. (1994). "Quality Standards for Antimicrobial Prophylaxis in Surgical Procedures," Clinical Infectious Diseases, 18, 422-427.

Dilawar, M. S., Khan, M. A., Zain-Ul-Abidin, Azeem, S., Majeed, K. A., Shahbaz, A. \& Khan, A. R. (2011). "Effect of Variable Degrees of Jejunal Resection upon Different Clinico-Biochemical Parameters in Dogs," Korean Journal of Veterinary Research, 51 (4) 309-311.

Fossum, T. W. (2007). Small Animal Surgery, Mosby, London, UK.

Gravante, G. \& Caruso, R. (2009). "Mechanical Bowel Preparation for Elective Colorectal Surgery: Is It Enough?," Journal of Gastrointestinal Surgery, 13 (10) 13921394. 
Gregory, S. (2003). 'Gastric Outflow Tract Disease,' Royal Vet College, London, UK.

Hayashi, M. S. \& Wilson, S. E. (2009). "Is There a Current Role for Preoperative NonAbsorbable Oral Antimicrobial Agents for Prophylaxis of Infection after Colorectal Surgery?," Surgical Infections, 10 (3) 285288.

Hinchey, E. J., Richard, 0. K. \& Prentis, J. (1983). "Metronidazole as a Prophylactic Agent in Wound Infection after Colon Surgery," Surgery, 93, 197-200.

Jain, N. C. (1986). 'Schalms Veterinary Haematology,' Philadelphia, USA.

Lalla, F. D. (2009). "Antimicrobial Prophylaxis in Colorectal Surgery: Focus on Ertapenem," Therapeutics and Clinical Risk Management, 5, 829-839.

Leaper, D. J. (1994). "Prophylactic and Therapeutic Role of Antibiotics in Wound Care," The American Journal of Surgery, 167, 155- 195.

Lindhagen, J., Hadziomerovic, A., Nordlung, S. \& Zbornik, J. (1981). "Comparison of Systemic Prophylaxis with MetronidazoleFosfomycin and MetronidazoleCephalothin in Elective Colorectal Surgery," Acta Chirurgica Scandinavica, 147 (4) 277283.

Mittermayer, H., Gross, C. \& Brucke, P. (1984). "Single Dose Cefuroxime/ Metronidazole versus Metronidazole Alone in Elective Colorectal Surgery," The American Surgeon, 50 (8) 418-423.

Nichols, R. L., Choe, E. U. \& Weldon, C. B. (2005). "Mechanical and Antibacterial Bowel Preparation in Colon and Rectal Surgery," Chemotherapy, 51 (10) 115-121.

Ono, S., Kato, S., Tanaka, T., Ishibiki, K., Kodaira, S. \& Abe, O. (1990). "Preoperative Oral Antimicrobial Bowel Preparations in Elective Colorectal Surgery," Nippon Geka Gakkai Zasshi, 91 (8) 972-979.

Parker, M. C., Ashby, E. C., Nicholls, M. W., Dowding, C. H. \& Brookes, J. C. (1985).
"Povidone-Iodine Bowel Irrigation before Resection of Colorectal Carcinoma," Annals of the Royal College of Surgeons of England, 67 (4) 227-228.

Playforth, M. J., Smith, G. M. R., Evans, M. \& Pollock, A. V. (1988). "Antimicrobial Bowel Preparation; Oral, Parenteral or Both," Diseases of the Colon \& Rectum, 31, 90-93.

Plumb, D. C. (2008). Veterinary Drug Handbook, Wiley-Blackwell Publishing Co, USA.

Rasmussen, L. (2002). Textbook of Small Animal Surgery, WB Saunders Co, Philadelphia, USA.

Rau, H. G., Mittelkotter, U., Zimmermann, A., Lachmann, A., Kohler, L. \& Kullmann, K. H. (2000). "Perioperative Infection Prophylaxis and Risk Factor Impact in Colon Surgery," Chemotherapy, 46, 353363.

Ross, D., Dijksman, L. M., Sondermeijer, B. M., Straaten, H. M. O., Dewit, L. T. \& Gerhards, M. F. (2009). "Perioperative Selective Decontamination of Digestive Tract (SDD) in Elective Colorectal Surgery," Journal of Gastrointestinal Surgery, 13 (10) 1839-1844.

Takesue, Y., Yokoyama, I., Akagi, O. S., Ohge, H., Murakami, Y., Sakashita, Y., Miyamoto, K., Vemura, K., Itaha, H. \& Matsuura, Y. (2000). "A Brief Course of Colon Preparation with Oral Antibiotics," Surgery Today, 30, 112-126.

Tavakoli, A., Bakhtiari, J. \& Khalaj, A. (2007). "Clinical Evaluation of Two Suture Pattern Techniques in Laparoscopic Gastrojejunostomy in Dog," Iranian Journal of Veterinary Surgery, 2 (2) 39-45.

Tobias, K. M. \& Ayres, R. (2006). "Key Gastrointestinal Surgeries: Intestinal Anastomosis," Veterinary Medicine, 1-6.

Van-Winkle, W. (1969). 'Tensile Strength of Wound and Factors That Influence It,' Surg Gynecol Obstet, 129, 819-842.

Rehan Rafique, Muhammad Nadeem Asi, Muhammad Saqib, Muhammad Hammad Hussain, Aqsa Mushtaq, Awais-ur-Rehman Sial, Hina Afroz and Muhammad Najiullah Khan (2013), International Journal of

Veterinary Medicine: Research \& Reports, DOI: 10.5171/2013.901239 
Wang, X. C., Gui, C. Q. \& Zheng, Q. S. (2003). "Combined Therapy of Allantoin, Metronidazole, Dexamethasone on the Prevention of Intra-Abdominal Adhesion in Dogs and Its Quantitative Analysis," World Journal of Gastroenterology, 9 (3) 568-571.

Weisman, D. L., Smeak, D. D., Birchard, S. J. \& Zweigart, S. L. (1999). "Comparison of a Continuous Suture Pattern with a Simple Interrupted Pattern for Enteric Closure in Dogs and Cats: 83 Cases (1991-1997)," Journal of the American Veterinary Medical Association, 214 (10) 1507-1510.

Woodfield, J. C., Van Rij, A. M., Pettigrew, R. A., Van Der Linden, A. J., Solomon, C. \& Bolt, D. (2003). "A Comparison of the Prophylactic Efficacy of Ceftriaxone and Cefotaxime in Abdominal Surgery," The American Journal of Surgery, 185 (1) 45-49. 